\title{
Estudo exploratório da Coesão em atletas juniores de futebol
}

Exploratory study of Cohesion in junior soccer athletes

\author{
Kauan Galvão Moräo \\ Laboratório de Estudos e Pesquisas em Psicologia do Esporte, \\ Universidade Estadual Paulista, Brasil \\ kauangm@hotmail.com \\ Renato Henrique Verzani \\ Laboratório de Estudos e Pesquisas em Psicologia do Esporte, \\ Universidade Estadual Paulista, Brasil \\ Guilherme Bagni \\ Laboratório de Estudos e Pesquisas em Psicologia do Esporte, \\ Universidade Estadual Paulista, Brasil

\section{Flávio Rebustini} \\ Laboratório de Estudos e Pesquisas em Psicologia do Esporte, \\ Universidade Estadual Paulista, Brasil \\ Afonso Antonio Machado \\ Laboratório de Estudos e Pesquisas em Psicologia do Esporte, \\ Universidade Estadual Paulista, Brasil
}

\section{RESUMEN:}

O objetivo foi investigar a coesão em atletas de futebol júnior. Foram analisados 162 atletas que participaram da Copa São Paulo. Para avaliar a coesão foi utilizado o Questionário de Ambiente de Grupo (QAG). Na análise estatística exploratória foram realizadas análises de estatísticas descritivas, análise multivariada de variância (MANOVA) e ANOVA para medidas repetidas ambas com post hoc teste de Bonferroni. Os resultados apontaram para níveis de coesão acima da média esperada tanto na dimensão "envolvimento pessoal" quanto "para a equipe" e a maior incidência de resultados importantes estatisticamente foram encontrados na comparação entre as posições de jogo. Parece ser possível indicar que as diferenças entre os níveis de coesão estão mais presentes quando analisamos a posição de jogo do que para idade, escolaridade e tempo de prática. Portanto, está mais centrada nas dinâmicas relacionais de grupo do que em características individuais dos atletas.

Palavras Chave: Coesão de grupo, Futebol, Psicologia do esporte.

\section{Abstract:}

The study aims to investigate the cohesion in junior soccer athletes. We analysed 162 athletes who participated in Copa São Paulo. To evaluate cohesion, we used the Questionário de Ambiente de Grupo (QAG). For the statistics analysis, descriptive analysis, multivariate analysis of variance (MANOVA) and ANOVA to repeated measures were conducted, both with Bonferroni' post hoc test. The results pointed out levels of cohesion above the expected average in dimensions such as "individual attraction" and "to group" and the higher incidence of important statically results were founded in comparison with game position. It might be possible to indicate that the differences between the cohesion levels are more present when we analyse the game position, rather than the age, education level and time of practice. Therefore, cohesion is more focused in group dynamics than in athletes' individual characteristics.

KEYWORDS: Group Cohesion, Soccer, Sport psychology. 


\section{INTRODUÇÃO}

Diversos são os fatores que podem acabar por facilitar a performance de cada equipe e de seus atletas. Alguns destes que merecem destaque são a interação entre os jogadores, a dinâmica do grupo, as normas e a comunicação, as lideranças e condições do grupo, sendo, portanto, importante ter olhares adequados em relação a quem lidera, ao isolamento e a coesão (Marques, 2016).

Trabalhar na intervenção torna necessário o conhecimento, os estudos e o entendimento sobre as dinâmicas envolvendo os atletas nos contextos esportivos, pois mesmo quando pensamos em atletas de modalidades conhecidas como individuais, temos grupos de treinamento (Machado, 1997).

Becker Junior (2008) e Marques (2016) destacam a existência de diferença com relação aos termos grupo e equipe. Para eles, treinar e competir juntos é o que torna os atletas um grupo, mas considera-se que cada um segue seus próprios objetivos. Já quando trata-se de uma equipe, temos que há um objetivo em comum, que prevalecerá em relação ao indivíduo, destacando a sobreposição da vontade do coletivo.

A coesão de grupo, de acordo com Carron (1988), é um processo dinâmico intimamente relacionado com a convergência do grupo em chegar aos objetivos e metas, mantendo-se assim juntos e reforçando a coesão. Para Gabarra (2008), a coesão é dependente de atletas que consigam se comunicar eficientemente, a ponto de haver compreensão mútua, melhorando o relacionamento a partir do reconhecimento grupal. Filho, Gershgoren, Basevitch e Tenenbaum (2014) verificaram que a coesão social de um grupo, em vez de individual, está positivamente associada a percepções do desempenho da equipe.

Alguns fatos que podem atrapalhar a coesão estão relacionados com a divergência enquanto aos objetivos, não definir ou a falta de aceitação em relação aos papéis de cada um, o olhar individualista, as trocas constantes de jogadores e problemas nas inter-relações entre os mesmos (Figueiras, 2007).

Quando um esportista expressa frustração com o mau desempenho de um companheiro de equipe, o resultado de tal ação pode levar o destinatário a pensar que é incapaz de contribuir com as metas da equipe, podendo acarretar em sentimento reduzido de coesão de equipe (Al-Yaaribi \& Kavussanu, 2017).

Cronin, Arthur, Hardy e Callow (2015) realizaram um estudo transversal com 381 atletas de diversas modalidades para examinar um modelo de mediação, segundo o qual a liderança transformacional está relacionada com a coesão direcionada a tarefa por meio do sacrifício. Os autores identificaram que o sacrifício tanto pessoal quanto em relação aos companheiros de equipe, mediaram as relações entre os comportamentos de liderança transformacional e a coesão das tarefas. Porém, foram notadas diferenças entre os sexos. Assim, os autores consideram que os treinadores devem se esforçar para mostrar comportamentos de liderança transformacional e como eles estão relacionados com o sacrifício pessoal e dos companheiros de equipe e a coesão direcionada a tarefa. Machado (2006) e Becker Junior (2008) salientam que o treinador possui um papel de destaque dentro de uma equipe esportiva, pois exerce um papel de poder e está presente tanto em treinamentos quanto em competições, o que pode ser beneficiado pela relação de proximidade com o responsável pela psicologia do esporte, reforçando questões como a melhoria na coesão da equipe, dentre outras.

Já Leo, González-Ponce, Sánchez-Miguel, Ivarsson e García-Calvo (2015), em um estudo com 351 atletas profissionais de futebol (homens e mulheres), participantes da segunda divisão masculina espanhola e da primeira divisão feminina espanhola, buscaram entender como as percepções de atletas quanto a conflitos de papéis, conflitos de equipe, ambiguidade de papéis e coesão de equipe podem explicar a eficácia coletiva ao longo da temporada. Uma das questóes confirmadas ressaltou que conflitos e coesão de grupo, durante uma temporada, explicam variações da eficácia coletiva.

A coesão de grupo no esporte é uma temática que apresenta diversas questões, até em relação a curiosidade do público em geral, pois tem-se a ideia de que coesão significa algo próximo da amizade. Na realidade podemos dividir a coesão em duas partes, segundo Spink e Carron (1992), temos então a coesão relacionada à tarefa e a coesão social, onde a primeira é como os integrantes do grupo trabalham em prol de objetivos 
comuns, enquanto a segunda relaciona-se mais com a questão da afinidade entre os membros. Para Cronin et al. (2015) a coesão direcionada a tarefa envolve a cooperação direcionada as metas de desempenho, tanto no treinamento quanto no ambiente competitivo

Portanto, muitas vezes acaba-se apenas visualizando a coesão social, especialmente devido à ampla divulgação da mídia nesses aspectos. Vale ressaltar que, assim, para compreender melhor a coesão, precisamos entendê-la como um sistema onde há a interação de diversas variáveis, tanto individuais como do grupo, conforme modelo desenvolvido por Carron (1982).

Como a coesão é uma variável de característica latente e, portanto, não pode ser diretamente medida, uma das maneiras de se avaliar essa questão é por meio de questionários, dentre os quais destacamos o Group Environment Questionnaire desenvolvido por Carron, Widmeyer e Brawley (1985) e que na língua portuguesa denomina-se Questionário de Ambiente de Grupo - QAG, de acordo com Gomes Neto (2011).

Com isso, o objetivo foi avaliar os níveis de coesão de atletas de futebol juniores por meio do Questionário de Ambiente de Grupo, verificando quais variáveis influenciam neste contexto e quais dimensões da coesão se sobressaem.

\section{Metodologia}

O estudo foi realizado com 162 atletas participantes da Copa São Paulo de Futebol Juniores, com idade média de 17,95 + 0,97 anos e tempo de prática médio de 9,02 + 3,21 anos. Com relação a escolaridade, 68,1\% possui ensino médio completo. Quanto a posição de jogo: 11,7\% (goleiros), 11,1\% (laterais), 16,7\% volantes, $15,4 \%$ (zagueiros), 22,2\% (meias), 18,5\% (atacantes) e 4,3\% não informaram sua posição.

Como instrumento foi utilizado o Questionário de Ambiente de Grupo (QAG), em sua versão adaptada para a língua portuguesa por Gomes Neto e Rosado em 2011, a qual foi derivada dos resultados obtidos na dissertação de Gomes Neto (2011). O questionário é composto por 12 itens divididos em 2 dimensões.

\section{AnÁlise Estatística}

Foram realizadas estatísticas descritivas minuciosas utilizando análises de tendência central (média, desvio padrão, mediana, média aparada, intervalos de confiança (IC) e intervalo interquartil (IQ)). Para a análise da distribuição dos dados foi testada a Assimetria e Achatamento e aplicado o Teste Kolmogorov-Smirnov (K-S) e o Teste de Normalidade Multivariada de Mardia.

Para avaliar os efeitos das variáveis independentes - idade, tempo de prática, nível educacional e posição de jogo foi utilizada Análise de Variância Multivariada (MANOVA). Utilizou-se a ANOVA para medidas repetidas para testar a existência de diferenças significativas na variância das médias entre os itens do instrumento. Para o post hoc test das análises de variância adotou-se a técnica de Bonferroni para controle dos erros do Tipo I. Foi aplicado um teste " $t$ " de amostra única para verificar o possível efeito de valores extremos sobre a média, o valor de referência foi o valor da média aparada. Em todas as técnicas foram adotados $\mathrm{p}<0,05$.

As análises foram efetuadas como SPSS 23.

\section{Resultados E Discussão}

A testagem da normalidade apontou para assimetria negativa violando a suposição de normalidade $(-1,96<$ assimetria/EP > 1,96); o K-S demonstrou que essas violações da normalidade são significativas $(\mathrm{p}<0,001)$ para todos os itens. O teste de normalidade multivariada também apontou violação da normalidade dos dados - $($ Mardia $(364)=41,15 ; \mathrm{p}<0,01)$. Contudo, não foram todos os itens que violaram a curtose, bem como, apenas os itens 3 e 4 apresentaram violação da homogeneidade (teste de Levene). Como a homogeneidade 
um dos pressupostos para uso de técnicas paramétricas e nem todos os itens tiveram violações plenas de distribuição dos dados e, finalmente, como as técnicas paramétricas tem um menor erro de medida associado e mais robustas, optamos pelo caminho das técnicas paramétricas.

Os resultados da análise descritiva (Tabela 1 ) indicaram que todas as questões do instrumento tiveram médias superiores à média esperada da escala (5), variando de 5,95 a 7,99. Todos os itens do instrumento quando testados com o Teste " $t$ " para uma única amostra tomando como referência a média esperada, apresentaram diferenças significativas $\mathrm{p}<0,01$, reforçando a evidência de que os dados não têm uma distribuição normal. As medianas também se posicionaram acima do centro da escala, podendo indicar uma aquiescência positiva de tendência de resposta dos participantes. A aquiescência pode demonstrar a constituição de uma tendência dos participantes em endossar itens (Leme \& Zanon, 2016).

A amplitude das respostas variou de 7 a 8 , indicando que foi utilizado praticamente todo o espectro de pontos da escala. Contudo, precisa-se verificar como os dados se posicionaram na pontuação da escala. Assim, como apontado na abertura dos resultados, todos os itens tiveram assimetria negativa, violando a suposição de normalidade $(-1,96<$ assimetria/EP > 1,96); o K-S demonstrou que essas violações da normalidade são significativas $(\mathrm{p}<0,001)$ para todos os itens. Quanto a curtose os itens 3, 4, 5, 7, 9, 10, 11 apresentaram valores de curva mesocúrtica $(-1,96<$ curtose/EP > 1,96) apresentando-se com distribuição normal para a curtose. Os itens 2, 6, 8 e 12 tiveram resultados leptocúrticos (curtose/EP > 1,96) e o item 1 teve configuração platicúrtica (curtose/EP < - 1,96). Ainda é possível atestar essa configuração do item 1 pela amplitude do intervalo interquartil que foi de 5 pontos, o que evidencia uma tendência de resposta mais espalhada na escala. Como comparação os itens leptocúrticos tiveram IQs de apenas 2 pontos, o que representa que 50\% das respostas distribuíram-se em apenas 2 pontos da escala.

As duas dimensões do instrumento "Desenvolvimento Pessoal" e "A Sua Equipe como um todo", apresentaram também assimetria negativa, mas com curtose mesocúrtica. Como cada uma das dimensões têm a possibilidade de se fazer 54 pontos, a média esperada é de 27 . Novamente, testamos a existência de diferença entre a média obtida com os atletas e o que se espera se a distribuição foi normal. Para as duas dimensões houve diferenças significativas entre a média esperada e a real. Para a dimensão "Desenvolvimento Pessoal" foi de $\mathrm{t}(161)=25,73 ; \mathrm{p}<0,001$ e para a dimensão "A sua equipe como um todo" foi de $\mathrm{t}(161)=24,18$; $\mathrm{p}<0,001$. O "Desenvolvimento Pessoal" e "A sua equipe como um todo" tiveram médias 42,91 + 7,92 e $41,82+7,72$, respectivamente. Não houve diferenças significativas entre as duas dimensões e a magnitude da diferença entre as médias pode ser considerada fraco ( $\mathrm{d}$ de Cohen = 0,13; IC 95\% [-0,09 a 0,35]). Este resultado indica que os interesses pessoais não estão se distanciando estatisticamente dos interesses voltados para a equipe e que os níveis de coesão são estatisticamente superiores à média esperada, portanto os atletas indicaram bons níveis de coesão para as duas dimensões.

De acordo com Paes, Machado, Berbetz e Stefanello (2016) normalmente as equipes esportivas costumam ser bastante orientadas para a tarefa. $O$ resultado envolvendo os interesses indica um alinhamento interessante, visto que a coesão pode direcionar para um ambiente que favoreça uma atuação coletiva satisfatória (Carron et al., 1985). 
TABELA 1

Estatística descritiva de tendência central e distribuição da amostra

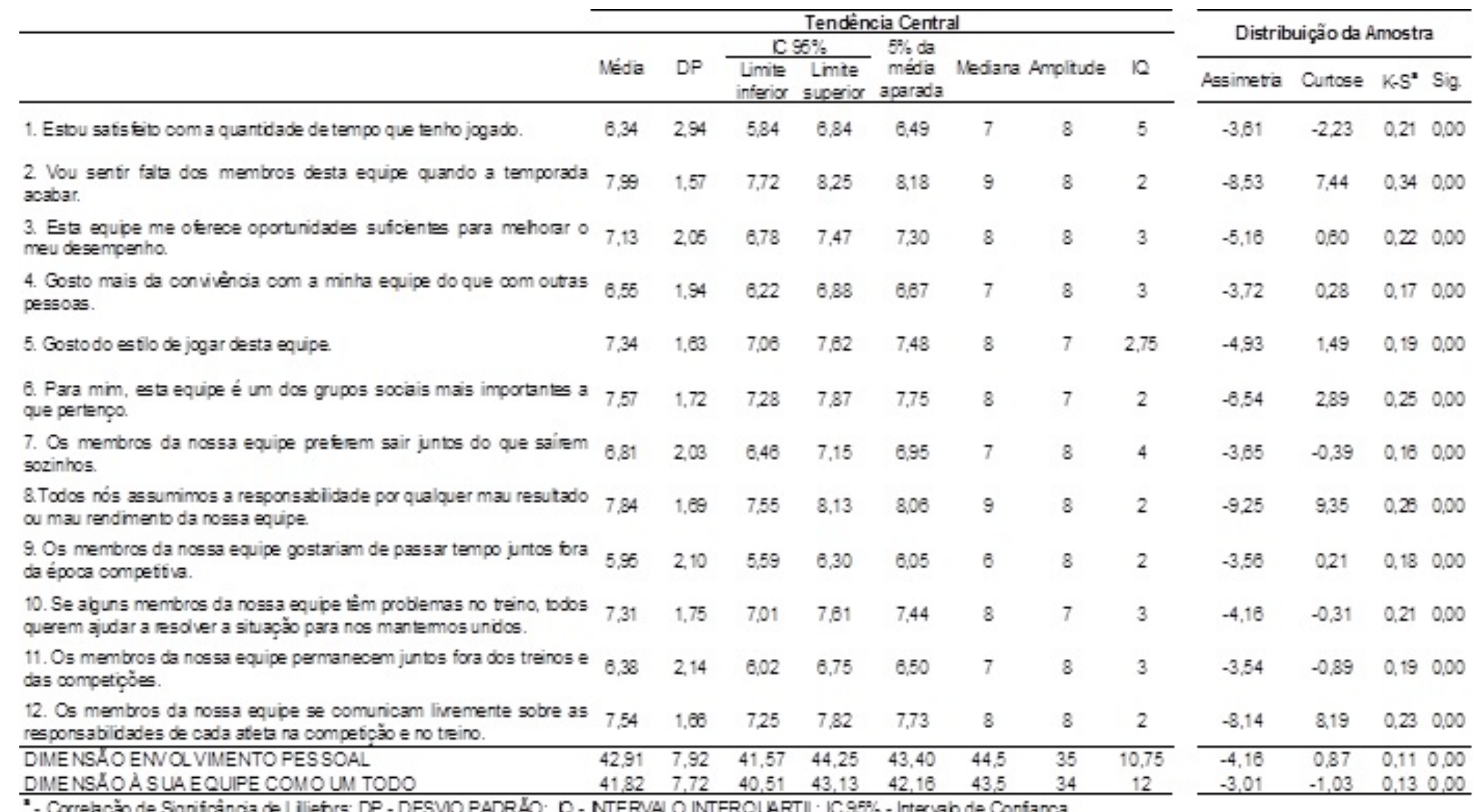

Além disso, em um estudo realizado por Balbim, Nascimento Junior e Vieira (2012), os esportistas que competem em uma divisão de maior nível e apresentam índices menores de coesão de grupo (tanto social, quanto para tarefa), possuem percepção positiva acerca dos fatores de estresse psicológico pré-competitivo direcionados ao desempenho.

Al-Yaaribi e Kavussanu (2017) realizaram um estudo com 272 atletas de modalidades coletivas, visando examinar se os comportamentos pró-sociais e antissociais dos companheiros de equipe estão relacionados à coesão da tarefa e ao burnout, além de analisar se o efeito positivo e negativo afetam essas relações. Foi constatado que o comportamento pró-social do companheiro de equipe previu positivamente a coesão da tarefa e negativamente o burnout, sendo que essas relações foram mediadas pelo efeito positivo. $\mathrm{O}$ padrão inverso de relacionamentos foi observado para o comportamento de companheiro de equipe antissocial que previu negativamente a coesão da tarefa e positivamente o burnout, tendo essas relações mediadas por afetos negativos. Tais constatações evidenciaram a importância de promover o comportamento pró-social e a redução dos comportamentos antissociais no esporte e destacar o papel do afeto na explicação das relações identificadas (Al-Yaaribi \& Kavussanu, 2017).

A coesão para tarefa no contexto do esporte pode ser considerada parte fundamental para que exista satisfação dos membros das equipes esportivas durante um campeonato (Spink, Nickel, Wilson \& Odnokon, 2005), pois quanto mais alto estiver o índice de envolvimento do esportista com os objetivos da equipe, também haverá maior nível de satisfação, acarretando em melhora do rendimento da equipe (Carron, Colman, Wheeler \& Stevens, 2002b; Chiocchio \& Essiembre, 2009).

Segundo Carron, Eys e Burke (2007), Nascimento Junior, Vieira, Sousa e Vieira (2011) e Karreman, Dorsch e Riemer (2009) é evidente que a confiança que os atletas possuem para alcançar os objetivos é influenciada pela interação dos mesmos dentro de seus times, salientando que a coesão grupal e a satisfação implicam em aspectos relevantes para o sucesso da equipe.

Caron, Bloom, Loughead e Hoffmann (2016) realizaram uma pesquisa que visava entender as percepções de atletas paralímpicos considerados líderes a respeito da liderança e da coesão de equipe. Dez atletas participaram do estudo por meio de entrevistas semiestruturadas. Os participantes relataram que eram 
responsáveis por motivar, apoiar e se comunicar com seus colegas de equipe e treinadores. Tais atletas também sentiam que possuíam papel de ajudar e incentivar seus companheiros a viver de forma independente. Também foi relatada a importância de organizarem reuniões sociais fora do cenário esportivo formal, buscando influenciar positivamente na coesão da equipe e melhorar o desempenho no esporte.

Eys et al. (2015) buscaram explorar as percepções da relação coesão-desempenho por treinadores que lideraram equipes de ambos os sexos. Foram realizadas entrevistas semiestruturadas com treinadores canadenses e alemães com experiências anteriores, onde lideraram equipes esportivas masculinas e femininas. Os resultados mostraram diferenças em relação ao sexo e ao envolvimento na prática. Assim, os autores afirmam que isso não significa que uma abordagem seja melhor ou mais "normal" do que outra, mas que se deve compreender melhor os indivíduos e o contexto para que se possa maximizar o rendimento esportivo.

Outro fato importante de ser ressaltado é que o sucesso esportivo pode ser resultante do envolvimento do esportista com questões relacionadas à coesão social da equipe (Carron et al., 2007; Carron et al., 1985), visto que tal coesão auxilia na melhora do rendimento esportivo e pode corroborar com a manutenção do atleta na equipe (Nathan et al., 2010). Contudo, em uma equipe, a coesão para a tarefa não pode ser muito menor que a coesão social, pois o envolvimento excessivo entre os membros do mesmo time pode gerar perda de foco, prejudicando a consecução das metas predeterminadas ao grupo (Hardy, Eys \& Carron, 2005). Neste sentido, estudos como os desenvolvidos por Ramzaninezhad e Keshtan (2009), Nascimento Junior, Souza e Vieira (2010) revelam que em atletas de alto rendimento, a busca pelos objetivos da equipe e a coesão para a tarefa são maiores que os níveis de coesão social, isto é, tais atletas consideram as relações sociais como secundárias no esporte.

De acordo com pesquisas anteriores (McLaren, Newland, Eys \& Newton, 2016) foi constatado que a coesão direcionada à tarefa foi inversamente associada ao conflito dentro do time (por exemplo, um indivíduo criticando seu companheiro de equipe quando comete erros), referindo-se a comportamentos negativos que podem prejudicar as relações interpessoais entre os envolvidos na situação (Ntoumanis \& Vazou, 2005). Além disso, jogadores de futebol e de basquete que percebem que seus companheiros de equipe apresentam comportamento antissocial em relação a eles durante uma partida, relataram sentir maior nível de raiva (AlYaaribi, Kavussanu \& Ring, 2016), enquanto as percepções dos atletas de interações sociais negativas foram associadas à ansiedade (Defreese $\&$ Smith, 2014). No esporte de alto rendimento os atletas apresentam bons níveis de coesão, principalmente direcionada à tarefa, colaborando com a queda de índices de ansiedade no momento de pré-competição (Prapavessis \& Carron, 1997).

Superada a análise geral inicial passamos a análise das variáveis de variância das médias. As análises foram realizadas para idade, posição, tempo de prática e nível educacional em relação ao teste.

Idade - $O$ primeiro estágio da Manova não apresentou diferenças significativas entre a variância das médias para idade e os itens do instrumento $\mathrm{F}(12,36)=1,11$; ns; $\lambda$ Wilks $=0,77 ; \eta 2$ parcial $=0,08$. O Teste de Levene apresentou violação da homogeneidade para os itens 3 e $4(\mathrm{~F}(3,158)=9,85$ e 4,24; $\mathrm{p}<0,01)$. No segundo estágio da Manova foi detectada diferença significativa entre as idades de 19 e 17 anos $(\mathrm{p}<0,05)$ para o Item 3 - "Esta equipe me oferece oportunidades suficientes para melhorar o meu desempenho". Os dados apontam que a idade nesse grupamento não afeta de forma estatisticamente importante os resultados do instrumento para as questões.

De acordo com Gomes e Machado (2010), os jovens atletas possuem maiores índices de coesão social, enquanto Carron, Bray e Eys (2002a) e Nascimento Junior et al. (2011) indicam que esportistas de elite (alto rendimento) geralmente apresentam níveis mais elevados de coesão voltada à tarefa. No estudo de Gomes Neto (2011), foi observado que atletas em formação (que eram considerados como os de até 18 anos) apresentavam maior nível de coesão em relação aos atletas com idades superiores, o que pode ter relação com os resultados encontrados, pois a maioria dos atletas estão dentro da faixa etária em questão e não foram encontradas diferenças significativas considerando a variância das médias para idade. 
Em uma pesquisa com centenas de jovens atletas de elite, com média de idade de aproximadamente 15 anos e praticantes de futebol e handebol, Benson, Siska, Eys, Priklerová e Slepicka (2016) encontraram relações positivas entre o desempenho esportivo e tanto da tarefa quanto formas sociais da coesão grupal. Isto é, os atletas apresentam níveis mais elevados de coesão da tarefa quando se apresentam bem, mas também têm percepção de maior união social, evidenciando a importância das relações interpessoais no desenvolvimento dos jovens através do esporte.

Escolaridade - $O$ primeiro estágio da Manova não apresentou diferenças significativas entre a variância das médias para Escolaridade e os itens do instrumento $\mathrm{F}(36,381)=1,18$; ns; $\lambda$ Wilks $=0,73$; $\eta 2$ parcial $=$ 0,09. O Teste de Levene apresentou violação da homogeneidade para os itens 1 e $9(F(3,140)=5,98$ e 4,00; $\mathrm{p}<0,01)$. No segundo estágio da Manova foi detectada diferença significativa para o item 1 "Estou satisfeito com a quantidade de tempo que tenho jogado" $(\mathrm{F}(3,140)=5,31 ; \mathrm{p}<0,05 ; \eta 2$ parcial $=0,06)$ entre os que tem nível superior incompleto e Ensino Médio Incompleto $(\mathrm{p}<0,05)$. Para o item 3, "Esta equipe me oferece oportunidades suficientes para melhorar o meu desempenho" $(\mathrm{F}(3,140)=4,89 ; \mathrm{p}<0,05 ; \eta 2$ parcial $=0,09)$ entre os que tem nível superior incompleto para Ensino Fundamental completo, Ensino Médio Incompleto e Ensino Médio Completo $(\mathrm{p}<0,05)$. Para o item 5, "Gosto do estilo de jogar desta equipe" $(\mathrm{F}(3,140)=4,40 ; \mathrm{p}$ $<0,05 ; \eta 2$ parcial $=0,08)$ entre os que tem nível superior incompleto para Médio incompleto e Fundamental Incompleto $(\mathrm{p}<0,05)$.

Comparando diversos níveis de escolaridade, Gomes Neto (2011) não conseguiu confirmar sua hipótese inicial de que quanto maior o nível de ensino dos atletas, menor seria a coesão encontrada entre eles, citando também em seu estudo a pouca atenção da literatura sobre este tipo de relação. Neste caso, também ficou constatada que não há diferença significativas envolvendo a variância das médias para escolaridade em itens da dimensão "interesses pessoais".

Tempo de prática - O primeiro estágio da Manova apresentou diferenças significativas entre a variância das médias para Tempo de prática e os itens do instrumento $\mathrm{F}(12,156)=1,35 ; \mathrm{p}<0,05 ; \lambda$ Wilks $=0,22$; $\eta^{2}$ parcial $=0,11$. O Teste de Levene apresentou violação da homogeneidade para os itens 1, 5, 7, 8 e 9 $(\mathrm{F}(13,140)=1,92$ a 3,16; $\mathrm{p}<0,01)$. No segundo estágio da Manova foi detectada diferença significativa para o item 8 , "Todos nós assumimos a responsabilidade por qualquer mau resultado ou mau rendimento da nossa equipe" $(\mathrm{F}(13,140)=2,49 ; \mathrm{p}<0,05 ; \eta 2$ parcial $=0,18)$ entre os que têm 11 anos de prática para os que têm $5,6,7,8,9,10$ e 12 anos ( $<<0,05)$. Para o item 12, "Os membros da nossa equipe se comunicam livremente sobre as responsabilidades de cada atleta na competição e no treino" $(F(13,140)=3,02 ; \mathrm{p}<0,05 ; \eta 2$ parcial $=0,21)$ entre os que têm 11 anos de prática para os que têm 3 a 14 anos.

Em uma meta-análise realizada por Carron et al. (2002b), a questão envolvendo a experiência dos competidores, foi considerada como um fator que não implicava fortemente na relação entre a coesão e o desempenho. Indo ao encontro do que ocorreu no estudo de Gomes Neto (2011), no qual foram encontradas diferenças significativas envolvendo os anos de prática, o presente estudo encontrou diferenças neste viés.

O estudo de Asamoah e Grobbelaar (2017), comparou equipes de futebol, acerca da coesão da equipe e diversas variáveis descritivas sendo utilizada como base a posição final alcançada num campeonato que envolveu equipes de 16 instituições. Os participantes responderam o questionário antes do início do campeonato de futebol da Universidade de Sudáfrica 2012 (USSA). Notou-se que as quatro melhores equipes apresentaram maior experiência anterior neste campeonato em relação as outras 12 equipes. A forte atração individual para o grupo foi vantajosa para o desempenho, enquanto níveis mais altos de integração de grupo foram associados com menor desempenho. Também foi observado por Carron et al. (2002a), que altos níveis de coesão implicam em positiva e significativa relação com o rendimento esportivo, sendo independente de fatores como o grau de experiência do atleta, modalidade que pratica, gênero, coesão voltada à tarefa ou social.

Posição de Jogo - O primeiro estágio da Manova apresentou diferenças significativas entre a variância das médias para posição de jogo e os itens do instrumento $\mathrm{F}(60,649)=1,38 ; \mathrm{p}<0,05 ; \lambda \mathrm{W}$ ilks $=0,57 ; \eta 2$ parcial 
= 0,06. O Teste de Levene apresentou violação da homogeneidade para os itens 6, 7 e $11(\mathrm{~F}(5,149)=2,36$ a 3,38; $\mathrm{p}<0,01)$. No segundo estágio da Manova, foi detectada diferença significativa para o item. Os meias e volantes apresentaram diferenças significativas na variância de suas médias para o item 3, "Esta equipe me oferece oportunidades suficientes para melhorar o meu desempenho." $(F(5,149)=2,55 ; \mathrm{p}<0,05 ; \eta 2$ parcial $=0,07)$ e para o item 4, "Gosto mais da convivência com a minha equipe do que com outras pessoas". Os goleiros apresentaram diferenças significativas para os laterais, meias, volantes e zagueiros $(\mathrm{F}(5,149)=2,80$; $\mathrm{p}<0,05 ; \eta 2$ parcial $=0,08)$ para o item 7 , "Os membros da nossa equipe preferem sair juntos do que saírem sozinhos". Ainda houve diferenças significativas entre os goleiros e laterais para a questão 9, "Os membros da nossa equipe gostariam de passar tempo juntos fora da época competitiva” $(F(5,149)=2,83 ; p<0,05 ; \eta 2$ parcial $=0,08)$. As questóes 4,7 e 9 tratam de convívio fora do ambiente de treinamentos e competições.

Quando analisamos os resultados das duas dimensões "Envolvimento Pessoal" e "A sua equipe como um todo" não foram encontradas diferenças significativas para as variáveis independentes idade, tempo de prática, posição e escolaridade.

Considerando a modalidade dos atletas estudados, temos que comumente as equipes têm orientação, muitas vezes, direcionadas a tarefa e que a coesão do grupo vai acabar interferindo no grau em que ocorrerá um trabalho em conjunto para atingir objetivos comuns (Carron et al., 1985). Ainda de acordo com estes autores levar em consideração a coesão social pode sinalizar o quanto que o companheirismo pode colaborar para a atuação coletiva. Provavelmente, algumas diferenças poderiam ser encontradas nas variáveis em questão caso os resultados fossem coletados em diversos momentos, visto que os resultados podem acabar impactando na coesão no decorrer das competições, de acordo com apontamentos envolvendo pesquisas com o gênero masculino (Carron \& Brawley, 2012).

$\mathrm{O}$ teste multivariado aplicado aos itens do questionário apresentou índice de $\mathrm{F}(11,151)=17,919 ; \mathrm{p}<$ 0,$001 ; \lambda \mathrm{Wilks}=0,434 ; \eta 2$ parcial $=0,56$. A esfericidade de $\mathrm{W}$ de Mauchly $(65)=0,12 ; \mathrm{p}<001$, atestando que existe diferença significativa entre as variâncias das diferenças (Field, 2013). O teste de efeitos entre os itens apresentou $\mathrm{F}(11,1771)=23,26 ; \mathrm{p}<001$, indicando a existência de diferenças significativas entre a variância das médias dos itens. Para atestar entre quais itens há diferenças significativas, passamos a análise do post hoc test de Bonferroni. A tabela 2 mostra quais itens apresentaram diferenças significativas para $p<0,05$. Das 66 possíveis interações, 35 apresentaram diferenças significativas. $\mathrm{O}$ item 2 "Vou sentir falta dos membros desta equipe quando a temporada acaba", é o item com a maior média $(7,99)$ e o item 9 "Os membros da nossa equipe gostariam de passar tempo juntos fora da época competitiva” com a menor média $(5,95)$. Interessante apontar a inconsistência entre os dois itens que tratam praticamente da mesma questão a convivência fora do ambiente de treinamentos e tem resultados opostos. Ambos os itens tiveram diferenças significativas para 8 itens. Seguidos pelos itens 4, "Gosto mais da convivência com a minha equipe do que com outras pessoas" com média de 6,55, e o item 11, "Os membros da nossa equipe permanecem juntos fora dos treinos e das competições” com média de 6,38, ambos os itens tiveram diferenças significativas com 7 outros itens. 
TABELA 2

Mapa de diferenças significâncias entre os itens do questionário

\begin{tabular}{|c|c|c|c|c|c|c|c|c|c|c|c|c|}
\hline item & 1 & 2 & 3 & 4 & 5 & 6 & 7 & 8 & 9 & 10 & 11 & 12 \\
\hline 1. E stou satisé ito $\infty \mathrm{m}$ a quantidade de tempo que tenho jog ado. & - & * & * & & * & * & & * & & * & & \\
\hline $\begin{array}{l}\text { 2. Vou sentir falta dos membros desta equipe quand o a temporad a } \\
\text { acabar. }\end{array}$ & & - & * & * & * & & * & & * & 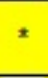 & * & \\
\hline $\begin{array}{l}\text { 3. E sta equipe me oferece oportunidades suficie ntes para melhorar } 0 \\
\text { meu de sempenho. }\end{array}$ & & & - & * & & & & & × & & * & \\
\hline $\begin{array}{l}\text { 4. Gosto mais da convivência com a minha equipe do que com outras } \\
\text { pessoas. }\end{array}$ & & & & - & * & & & * & & * & & * \\
\hline 5. Gosto do estilo de jogar desta equipe. & & & & & - & & & & * & & * & \\
\hline $\begin{array}{l}\text { 6. P ara mim, esta equipe é um dos grupos socia is mais importa ntes a } \\
\text { que perte nco. }\end{array}$ & & & & & & - & * & & * & & * & \\
\hline $\begin{array}{l}\text { 7. Os me mbros da nossa equipe preferem sair juntos do que sairem } \\
\text { sozinhos. }\end{array}$ & & & & & & & - & * & * & & & * \\
\hline $\begin{array}{l}\text { 8. Todos nós assumimos a responsa bilidade por qua lquer mau re sultado } \\
\text { ou mau rendimento da nossa equipe. }\end{array}$ & & & & & & & & - & 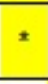 & & * & \\
\hline $\begin{array}{l}\text { 9. Os me mbros da nossa equipe gostariam de passar tempo juntos fora } \\
\text { da época competitiva. }\end{array}$ & & & & & & & & & - & ะ & & $\star$ \\
\hline $\begin{array}{l}\text { 10. Se a lguns me mbros da nossa equipe têm problem as no treino, todos } \\
\text { querem a judar a re solver a situacão para nos mantermos unidos. }\end{array}$ & & & & & & & & & & & * & \\
\hline $\begin{array}{l}\text { 11. Os membros da nossa equipe permanecem juntos fora dos treinos e } \\
\text { das competicôes. }\end{array}$ & & & & & & & & & & & & * \\
\hline $\begin{array}{l}12 . \text { Os membros da nossa equipe se comunicam livem ente so bre as } \\
\text { responsabilidades de cada atleta na competicão e no treino. }\end{array}$ & & & & & & & & & & & & \\
\hline
\end{tabular}

\section{Conclusões}

Os resultados apontaram para níveis de coesão acima da média esperada para o teste, indicando escores satisfatórios de coesão tanto na dimensão "envolvimento pessoal" quanto "para a equipe". A idade não parecer ser uma variável que interfere na coesão de um grupo. Para escolaridade aparecerem diferenças significativas para alguns itens do questionário entre os maiores níveis educacionais e os itens 1, 3 e 5 do instrumento, todos eles pertencentes a dimensão "interesses pessoais". Estes resultados diferem parcialmente dos encontrados no estudo original de Gomes (2011) em que não foram encontradas diferenças estatísticas para o nível de escolaridade.

Quanto à posição de jogo houveram diferenças entre as posições de meias e volantes relativas à questão para tarefa. Para os goleiros os níveis de coesão foram mais baixos que outras posições. O que pode evidenciar a necessidade de um trabalho diferenciado para criar uma relação maior de coesão entre os goleiros e as outras posições de jogo. Para essa variável independente três dos itens 4, 7 e 9 tratam da relação dos atletas fora do ambiente de jogo, o que pode indicar que os goleiros não estão integrados a estas dinâmicas.

Para o tempo de prática foram encontradas diferenças para os atletas que tinham 11 anos de prática em relação aos atletas com menos tempo de prática. Para a posição, há evidências fortes da diferença nos índices de coesão entre os goleiros e os demais membros das equipes. Isto pode ser explicado pelo modelo de treinamento, pois os goleiros essencialmente formam um grupo a parte na estrutura de treinamento, por vezes, treinam isolados dos demais atletas. Esta estrutura pode contribuir para que os goleiros tenham menores índices de coesão.

Desta forma, parece ser possível indicar que as diferenças entre os níveis de coesão estão mais presentes quando analisamos a posição de jogo do que para idade, escolaridade e tempo de prática. Portanto, está mais centrada nas dinâmicas relacionais de grupo do que em características individuais dos atletas. 


\section{REFERENCIAS}

Al-Yaaribi, A., \& Kavussanu, M. (2017). Teammate Prosocial and Antisocial Behaviors Predict Task Cohesion and Burnout: The Mediating Role of Affect. Journal of Sport \& Exercise Psychology, 39(3), 199-208. doi:https:// doi.org/10.1123/jsep.2016-0336

Al-Yaaribi, A., Kavussanu, M., \& Ring, C. (2016). Consequences of prosocial and antisocial behavior for the recipient. Psychology of Sport and Exercise, 26, 102-112. doi:https://doi.org/10.1016/j.psychsport.2016.06.012

Asamoah, B., \& Grobbelaar, H. W. (2017). Team cohesion and performance during a university soccer championship: two sides of the coin. South African Journal for Research in Sport, Physical Education and Recreation, 39(1), 17-31.

Balbim, G. M., Nascimento Junior, J. R. A., \& Vieira, L. F. (2012). Análise do nível de coesão de grupo e do estresse psicológico pré-competitivo de atletas adultos de voleibol. Rev Bras Cineantropom Desempenho Hum, 14(6), 704-712. doi:http://dx.doi.org/10.5007/1980-0037.2012v14n6p704

Becker Junior, B. (2008). Manual de psicologia do esporte e do exercício. Porto Alegre-RS: Novaprova.

Benson, A. J., Siska, P., Eys, M., Priklerová, S., \& Slepicka, P. (2016). A prospective multilevel examination of the relationship between cohesion and team performance in elite youth sport. Psychology of Sport and Exercise, 27, 39-46. doi:https://doi.org/10.1016/j.psychsport.2016.07.009

Caron, J. G., Bloom, G. A., Loughead, T. M., \& Hoffmann, M. D. (2016). Paralympic Athlete Leaders' Perceptions of Leadership and Cohesion. Journal of Sport Behavior, 39(3), 219-238.

Carron, A. V. (1982). Cohesiveness in sport groups: interpretations and considerations. Journal of Sport Psychology, 4(2), 123-138. doi:https://doi.org/10.1123/jsp.4.2.123

Carron, A. V. (1988). Group dynamics in sport. London: Spody.

Carron, A. V., \& Brawley, L. R. (2012). Cohesion conceptual and measurement issues. Small Group Research, 43(6), 726-743. doi:https://doi.org/10.1177/1046496412468072

Carron, A. V., Bray, S. R., \& Eys, M. A. (2002a). Team cohesion and team success in sport. J Sports Sci, 20(2), 119-126. doi:https://doi.org/10.1080/026404102317200828

Carron, A. V., Colman, M. M., Wheeler, J., \& Stevens, D. (2002b). Cohesion and performance in sport: A metaanalysis.J Sport Exerc Psychol, 24(2), 168-188. doi:https://doi.org/10.1123/jsep.24.2.168

Carron, A. V., Eys, M. A., \& Burke, S. M. (2007). Team cohesion: nature, correlates, and development. In S. Jowett, \& D. Lavallee (Orgs.). Social psychology in sport (pp. 91-102). Champaign: Human Kinetics.

Carron, A. V., Widmeyer, W. N., \& Brawley, L. R. (1985). The Development of an Instrument to Assess Cohesion in Sport Teams: The Group Environment Questionnaire. Journal of Sport Psychology, 7(3), 244-266. doi:http s://doi.org/10.1123/jsp.7.3.244

Chiocchio, F., \& Essiembre, H. (2009). Cohesion and performance: a meta-analytic review of disparities between project teams, production teams, and service teams. Small Group Research. 40(4), 382-420. doi:https://doi.or $\mathrm{g} / 10.1177 / 1046496409335103$

Cronin, L. D., Arthur, C. A., Hardy, J., \& Callow, N. (2015). Transformational Leadership and Task Cohesion in Sport: The Mediating Role of Inside Sacrifice. Journal of Sport \& Exercise Psychology, 37(1), 23-36. doi:https ://doi.org/10.1123/jsep.2014-0116

Defreese, J. D., \& Smith, A. L. (2014). Athlete social support, negative social interactions, and psychological health across a competitive sport season. Journal of Sport \& Exercise Psychology, 36(6), 619-630. doi:https://doi.or g/10.1123/jsep.2014-0040

Eys, M., Evans, M. B., Martin, L. J., Ohlert, J., Wolf, S. A., Bussel, M. V., \& Steins, C. (2015). Cohesion and Performance for Female and Male Sport Teams. The Sport psychologist, 29(2), 97-109. doi:http://dx.doi.org/1 $0.1123 /$ tsp.2014-0027

Field A. (2013). Discovering Statistics Using SPSS. Londres: Sage.

Figueiras, O. G. (2007). Cohesión de equipo. Lecturas Educación Física y Deportes, 12(114). Recuperado de http://w ww.efdeportes.com/efd114/cohesion-de-equipo.htm 
Filho, E., Gershgoren, L., Basevitch, I., \& Tenenbaum, G. (2014). Profile of high-performing college soccer teams: An exploratory multi-level analysis. Psychology of Sport and Exercise, 15(5), 559-568. doi:https://doi.org/10.1016/ j.psychsport.2014.05.008

Gabarra, D. O. (2008). O desenvolvimento do papel profissional e o trabalho em grupo para a ampliação e qualidade do atendimento. Revista Brasileira de Psicodrama, 16(1), 27-44.

Gomes Neto, D. A. (2011). Teste à validade do questionário de coesão de grupo em atletas de futebol (Dissertação de Mestrado). Universidade Técnica de Lisboa, Faculdade de Motricidade Humana, Lisboa, Portugal.

Gomes, A. R., \& Machado, A. A. (2010). Liderança, coesão e satisfação em equipes de voleibol portuguesas: indicações da investigação e implicações práticas. In M. R. F. Brandão, \& A. A. Machado (Orgs.). O voleibol e a psicologia do esporte (pp. 187-218). São Paulo: Editora Atheneu.

Hardy, J., Eys, M. A., \& Carron, A. V. (2005). Exploring the Potential Disadvantages of High Cohesion in Sports Teams. Small Group Research, 36(2), 166-187. doi:https://doi.org/10.1177/1046496404266715

Karreman, E., Dorsch, K., \& Riemer, H. (2009). Athlete Satisfaction and Leadership: Assessing Group-Level Effects. Small Group Research, 40(6), 720-737. doi:https://doi.org/10.1177/1046496409346450

Leme, K. E. F., \& Zanon, C. (2016). Criação de um índice de aquiescência para a escala de satisfação de vida. Anais do Encontro de Pós-graduação. USF, Bragança Paulista, Brasil. Recuperado de https://www.usf.edu.br/ic_2016/pdf/ic/psicologia/CRIACAO-DE-UM-INDICE-DE-AQUIESCENCI A-PARA-A-ESCALA-DE-SATISFACAO-DE--VIDA.pdf

Leo, F. M., González-Ponce, I., Sánchez-Miguel, P. A., Ivarsson, A., \& García-Calvo, T. (2015). Role ambiguity, role conflict, team conflict, cohesion and collective efficacy in sport teams: A multilevel analysis. Psychology of Sport and Exercise, 20, 60-66. doi:https://doi.org/10.1016/j.psychsport.2015.04.009

Machado, A. A. (2006). Psicologia do esporte: da educação física escolar ao esporte de alto nivel. Rio de Janeiro: Guanabara Koogan.

Machado, A. A. (1997). Psicologia do esporte: temas emergentes. Jundiaí: Ápice.

Marques, M. G. (2016). O teste sociométrico como instrumento de coesão de uma equipe de futebol. In M. R. F. Brandão, \& A. A. Machado (Orgs.). Competências psicológicas no esporte infanto-juvenil. (pp. 155-168). Várzea Paulista: Fontoura.

McLaren, C.D., Newland, A., Eys, M., \& Newton, M. (2016). Peer-initiated motivational climate and group cohesion in youth sport. Journal of Applied Sport Psychology, 29(1), 88-100. doi:https://doi.org/10.1080/10413200.201 6.1190423

Nascimento Junior, J. R. A., Vieira, L. F., Sousa, E. A., \& Vieira, J. L. L. (2011). Nível de satisfação do atleta e coesão de grupo em equipes de futsal adulto. Rev Bras Cineantropom Desempenho Hum, 13(2), 138-144. doi:https:/ /doi.org/10.5007/1980-0037.2011v13n2p138

Nascimento Junior, R. A., Souza, E. A., \& Vieira, L. F. (2010). Avaliação da percepção de coesão de grupo de equipes profissionais de futsal do Estado do Paraná. Lecturas Educación Física y Deportes. 15(151), 1-8. Recuperado de h ttp://www.efdeportes.com/efd151/coesao-de-grupo-de-equipes-profissionais-de-futsal.htm

Nathan, S., Birouste, A. B., Evers, C., Kemp, L., Mackenzie, J., \& Henley, R. (2010). Social cohesion through football: a quasi-experimental mixed methods design to evaluate a complex health promotion program. BMC Public Health, 10(1), 587-590. doi:https://doi.org/10.1186/1471-2458-10-587

Ntoumanis, N., \& Vazou, S. (2005). Peer motivational climate in youth sport: Measurement development and validation. Journal of Sport \& Exercise Psychology, 27(4), 432-455. doi:https://doi.org/10.1123/jsep.27.4.432

Paes, M. J., Machado, T. A., Berbetz, S. A., \& Stefanello, J. M. F. (2016). Frequência, intensidade e direção da ansiedade e sua relação com a coesão grupal em uma equipe de voleibol infanto-juvenil masculina. Revista Brasileira de Psicologia do Esporte, 6(3), 46-56.

Prapavessis, H., \& Carron, A. V. (1997). Sacrifice, cohesion, and conformity to norms in sport teams. Group Dynamics:Theory, Research, and Practice, 1(3), 231-240. doi:http://dx.doi.org/10.1037/1089-2699.1.3.231

Ramzaninezhad, R., \& Keshtan, M. H. (2009). The relationship between coach's leadership styles and team cohesion in Iran football clubs professional league. Brazilian Journal of Biomotricity, 3(2), 111-120. 
Spink, K. S., \& Carron, A. V. (1992). Group Cohesion and Adherence in Exercise Classes. Journal of Sports and Exercise Psychology, 14(1), 78-86, 1992. doi:https://doi.org/10.1123/jsep.14.1.78

Spink, K. S., Nickel, D., Wilson, K., \& Odnokon, P. (2005). Using a multilevel approach to examine the relationship between task cohesion and team task satisfaction in elite ice hockey players. Small Group Research, 36(5), 539-55. doi:https://doi.org/10.1177/1046496405275229 